\title{
An Interactive Visual Data Acquisition Tool for Multistage Care and Risk Evaluation of Pressure Ulcer Based on REDCap
}

\begin{abstract}
Huimeng LIU, ${ }^{\mathrm{a}, 1}$, Yutong WANG ${ }^{\mathrm{a}}$, Suixia CAO ${ }^{\mathrm{a}}$, Jingchun LIU ${ }^{\mathrm{a}}$ and Baibing MI ${ }^{\mathrm{a}, 2}$ ${ }^{a}$ Department of Epidemiology and Biostatistics, School of Public Health \& Global Health Institute, Xi'an Jiaotong University Health Science Center
\end{abstract}

Keywords. Pressure ulcer prevention; Electronic data Acquisition Tool; REDCap

\section{Introduction}

Pressure Ulcer (PU) is a frequently occurring health problem throughout the world both in clinical settings and home care[3]. Nursing skills and a holistic multi-stage (including clinical settings and home care) risk assessment can benefit PU warning, prevention, and individualized treatment.[1]. Our study aims to develop an interactive visual data acquisition tool for multi-stage care and risk evaluation of PU, which may (1) help nurses acquire more accurate information in clinical settings; (2) be convenient and understandable for patients (or their caregivers) to pre-warn the risk of PU prevalence during home care.

\section{Method}

REDCap is an open-source platform for building and managing online surveys and datasets. It contains central server (works on Windows or Android) electronic tablets such as smartphones. One Chinese version platform was operated and maintained by the School of Public Health Xi'an Jiaotong University.

In this tool, the intensity of pain is evaluated with Face Pain Scale (FPS) [2]. We used Braden Scale and referred to EPUAP practice guidelines [4] to assess the risk and Stage of PU, respectively [5]. We use REDCap external module, Custom Image Map, to visualize Braden Scale. A calculation field and "Branching Logic" function was set to automatically calculate the total risk score and warn the risk of PU.

\footnotetext{
${ }^{1}$ Corresponding Author, Huimeng Liu, Department of Epidemiology and biostatistics School of Public Health \& Global Health Institute Xi'an Jiaotong University Health Science Center; E-mail: xjtu.lhm@stu.xjtu.edu.cn.

${ }^{2}$ Corresponding Author, Baibing Mi, Department of Epidemiology and biostatistics School of Public Health \& Global Health Institute Xi'an Jiaotong University Health Science Center; E-mail: xjtu.mi@stu.xjtu.edu.cm.
} 


\section{Results}

This interactive visual PU risk evaluation tool can use for multi-stage caring, including clinical settings and home care (scan the QR code (Figure $1 \mathrm{G}$ ) to get access to the survey). Reference pictures can help nurses get more accurate information on the PU stage and help them get trained and improve skills on category identification (Figure 1D). For home care patients, the risk evaluation includes the location of PU (Figure 1A), pain evaluation (including pain intensity and pain type) (Figure 1B and C), visual Braden Sale (Figure 1E). After self-assessment, they will get a total score and evaluated risk of PU (Figure 1F). Besides, doctors and nurses can also see the dynamic changes of evaluation results through the central server and modify prevention measures or treatment timely

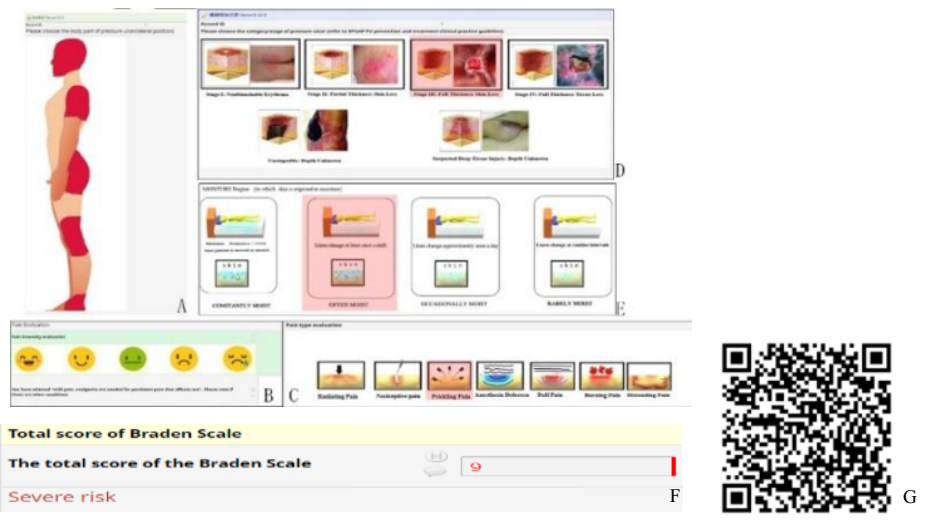

Figure 1. Interactive visual data acquisition tool for multi-stage care and risk evaluation of PU.

\section{Conclusions}

This interactive and visual tool will help nurse education, PU risk assessment, and take effective interventions timely.

\section{References}

[1] S. Bergquist-Beringer, L. Dong, J. He, and N. Dunton, Pressure ulcers and prevention among acute care hospitals in the United States, Jt Comm J Qual Patient Saf 39 (2013), 404-414.

[2] K.A. Herr, P.R. Mobily, F.J. Kohout, and D. Wagenaar, Evaluation of the Faces Pain Scale for use with the elderly, Clin J Pain 14 (1998), 29-38.

[3] B. Hiser, J. Rochette, S. Philbin, N. Lowerhouse, C. Terburgh, and C. Pietsch, Implementing a pressure ulcer prevention program and enhancing the role of the CWOCN: impact on outcomes, Ostomy Wound Manage 52 (2006), 48-59.

[4] National Pressure Ulcer Advisory Panel European Pressure Ulcer Advisory Panel and Pan Pacific Pressure Injury Alliance, Prevention and Treatment of Pressure Ulcers: Clinical Practice Guideline. Emily Haesler (Ed.), in, Osborne Park, Western Australia, 2014.

[5] M. Wei, L. Wu, Y. Chen, Q. Fu, W. Chen, and D. Yang, Predictive Validity of the Braden Scale for Pressure Ulcer Risk in Critical Care: A Meta-Analysis, Nurs Crit Care 25 (2020), 165-170. 